\title{
Multi-scale simulations of cardiac electrophysiology and mechanics using the University of Tokyo heart simulator
}

\author{
Seiryo Sugiura ${ }^{1, *}$, Takumi Washio ${ }^{1, *}$ Asuka Hatano ${ }^{1}$, Junichi Okada ${ }^{1}$, Hiroshi Watanabe ${ }^{1}$, \\ Toshiaki Hisada $^{1}$ \\ ${ }^{1}$ Department of Human and Engineered Environmental Studies, Graduate School of Frontier \\ Sciences, The University of Tokyo, \\ Kashiwanoha 5-1-5, Kashiwa-shi, Chiba, Japan 277-8563 \\ * These authors equally contributed to this work. \\ e-mail: Asuka Hatano \\ asuka-h@sml.k.u-tokyo.ac.jp \\ Takumi Washio \\ washio@sml.k.u-tokyo.ac.jp \\ Junichi Okada \\ okada@sml.k.u-tokyo.ac.jp \\ Hiroshi Watanabe \\ nabe@sml.k.u-tokyo.ac.jp \\ Toshiaki Hisada \\ hisada@mech.t.u-tokyo.ac.jp
}

Corresponding author: Seiryo Sugiura, M.D., Ph.D.

Address: Department of Human and Engineered Environmental Studies, Graduate School of Frontier Sciences, The University of Tokyo, Kashiwanoha 5-1-5, Kashiwa, Chiba, Japan 277-8563

e-mail: sugiura@k.u-tokyo.ac.jp

tel: $\quad+81358418393$

fax: $\quad+81358416376$ 


\begin{abstract}
The importance and need for an integrative mathematical modeling approach in the biological and medical fields is currently well recognized. Such an approach is crucial to understanding the complexity of hierarchical biological systems increasingly revealed by active researches in molecular and cellular biology. Particularly in cardiac functioning, modeling must cover such diverse phenomena as solid mechanics, fluid dynamics, electricity and biochemistry. Recent advancements in computational science and the development of high-performance computers have enabled the creation of multi-scale, multi-physics simulation heart models using the finite element method. Although whole heart or ventricular models of electrophysiology involving electro-mechanics with or without blood flow dynamics have been reported, to our knowledge no single model has yet succeeded in completely reproducing the behavior of the heart from the subcellular to whole organ levels. In this article, we present a brief methodology-focused review on some of the essential components for multi-scale, multi-physics heart modeling. A perspective of heart modeling in the era of high performance computing is also presented.
\end{abstract}

Key words: heart model, computer simulation, electrophysiology, cooperativity, fluid-structure interaction, cardiac metabolism 


\section{Introduction}

Since the pioneering work of Noble (Noble, 1960), numerical simulation has been recognized as a powerful and indispensable tool for understanding the electrophysiology of the heart. Electrophysiology modeling is now applied to diverse cell types from many animal species in both normal and diseased conditions (Beeler and Reuter, 1977; Luo and Rudy, 1994 ; Stewart et al., 2009 ; Ten Tusscher et al., 2004; Ten Tusscher and Panfilov, 2006; Winslow et al., 2001). The explosion of knowledge obtained at the molecular level of ion channel activity necessitates the development of more detailed modeling (Silva et al., 2009). Several integrative approaches to coupling these cell models have been proposed, which have enabled cardiac electrical activity at the tissue or organ level to be reasonably well-simulated (Noble, 2002; Vigmond et al., 2009). A similar history is found for the simulation of mechanical cardiac muscle activity, typically based on the cross-bridge model proposed by Huxley to describe skeletal muscle contraction (Huxley, 1957). In simulating the energy metabolism of heart muscle contraction, the cross-bridge model has been modified to account for the behavior of the cardiac myosins of distinct kinetic properties, and/or is coupled with ATP hydrolysis. Coupling of the cross-bridge model with the electrophysiology model via the inclusion of $\mathrm{Ca}^{2+}$ dependent regulatory mechanisms has enabled the excitation-contraction coupling process to be accurately simulated at the cellular level (Hunter et al., 1998; Landesberg and Sideman, 1994; Negroni and Lascano, 2008; Rice et al., 2008). From a clinical perspective, organ level simulation of the heart has significantly contributed to our understanding of hemodynamics (Beyar and Sideman, 1984; Sunagawa and Sagawa, 1982). Even so, earlier attempts to link these two levels failed to attract the broad-ranging interests of basic and clinical cardiology researchers. Today, the importance and need for integrative approaches by mathematical modeling is well recognized in the biological and medical fields (Hunter and Borg, 2003). As emphasized by Hunter et al. (Hunter and Borg, 2003), such needs stem from the complexity of hierarchical biological systems, which is being increasingly revealed by molecular and cellular biology studies. For example, imaging information on structure and function not only allow but force us to create a spatially distributed parameter model with realistic morphologies realized at levels from subcellular to whole organ. In the case of cardiac function, modeling must further embrace the diverse phenomena governed by the different physical principles involved, such as solid mechanics, fluid dynamics, electricity and biochemistry. Molecular simulation is expected to play a large role in future studies of this nature. Fortunately, recent advancements in computational science and the development of high-performance computers have enabled multi-scale, 
multi-physics simulation models of the heart to be constructed, using the finite element method (Lee et al., 2009; Noble, 2002; Trayanova, 2011). Whole heart or ventricular models of electrophysiology and electro-mechanics with or without flow dynamics in the chambers have been widely reported. However, to our knowledge, no single model has successfully reproduced the behavior of the heart at scales ranging from the subcellular to the whole organ.

In this article, we review and discuss some of the essential components and methodologies required for the development of a multi-scale, multi-physics heart simulation that integrates the molecular and cellular mechanisms of cardiac excitation, and contraction to reproduce the organ level electrophysiology and hemodynamics. Such a model could not only facilitate our understanding on how changes in molecular and cellular elements affect the macroscopic behavior of the heart, but may also supply detailed information on the micro-environment in which these elements are embedded in both physiological and pathological conditions.

In the concluding section, we present our own perspective on the potential use of super-computers for multi-scale simulation.

\section{ECG simulation}

A key aim of multiscale heart simulation is to establish the relation between the ion currents in the cell and the clinically obtained body surface ECG. Establishing this relationship is of paramount importance to clinical cardiology since it would improve clinical outcomes and pave the way for custom designed treatments. Because the basic framework for the development of the whole heart model of electrophysiology is well established, we here focus on reproducing the body surface potential map, or ECG, based on the cell model of electrophysiology. An alternative approach is to solve the inverse problem; however, due to the ill-posed nature of this problem, a reliable solution is currently available only for the cardiac surface (Ramanathan et al., 2004).

To obtain the body surface potential we need to solve the potential distribution in the model torso created by the electrical activity of the heart. Such simulations require huge computational resources, thus computational efficiency becomes a prime concern. Xue et al., assuming isotropic conductivity in the torso, coupled the bidomain model-based finite element method (FEM) to the boundary element method (BEM) in their study of T-wave morphology (Xue et al., 2009). In this approach, the FEM solution of the potential distribution in the anisotropic myocardium is first obtained. Using the potential on the heart boundary as the source term, the BEM problem is then solved to obtain the potential on the torso surface. This is an efficient way to solve the forward 
problem, but the advantage is lost if applied to torsos with numerous isotropic tissue types (Fischer et al., 2000). Furthermore, this is not a dynamic approach based on obtained source patterns independent of the torso.

The significant effect of different tissue conductivities on ECG morphology has also been noted (Keller et al., 2010). In our previous study, we also confirmed the effects of heterogeneity in torso conductivity (Washio et al., 2010). Taken together, realistic modeling of the torso allowing different tissue conductivities is a prerequisite of patient specific ECG simulation. In this context, an FEM model of the realistic torso has been applied to the simulation of body surface potential. However, most ECG simulations have, to date, adopted a two-step static approach consisting of the mono-domain calculation of membrane potential of the heart domain and FEM solution of the torso following interpolation of membrane potential on the finite elements (Farina et al., 2009; Keller et al., 2011; Keller et al., 2010). Although the membrane potentials obtained by the monodomain model and the bidomain calculation do not appreciably differ (Potse et al., 2006), this finding may be undermined by further analysis. When applied to defibrillation, the bidomain model coupled to a realistic multi-tissue torso should definitely be subject to further analysis.

We have developed a parallel multilevel technique for the dynamic solution of the bidomain equation for heart electrophysiological analysis coupled with the torso (Washio et al., 2010). For the FEM solution of the discretized bidomain equations, we adopt a composite mesh composed of a fine (local) voxel mesh around the heart and a coarse (global) voxel mesh covering the torso because fine resolution is unnecessary for the extracellular domain outside the heart (Fig. 1). To conserve the electric currents in the local-global solution process, the Lagrangian multiplier method for the constraints at the interface of the local and global meshes is applied. A novel treatment to include the Purkinje fiber network in the local-global multi-grid algorithm is also introduced.

Using this technique, we simulated the human body surface ECG and analyzed the cellular basis of ECG wave form (Okada et al., 2011). The morphology of the human heart with a conduction system embedded in the torso model was based on computerized tomography (CT) scan data. Informed consent for the use of clinical data was granted by the Institutional Ethics Committee. In describing the electrophysiological properties, we adopted (for the appropriate elements) the ventricular myocyte model of ten Tusscher and Panfilov (Ten Tusscher and Panfilov, 2006) and the Purkinje fiber model of Stewart et al. (Stewart et al., 2009). The network of the conduction system is modeled by one-dimensional elements and comprises a free-running (insulated) part connecting the atrioventricular node to the sites of earliest 
activation, and a network spreading from these sites along the endocardial surface. Network distribution was adjusted to reproduce the activation sequence of the human heart reported by Durrer et al. (Durrer et al., 1970). On the other hand, the distribution of different cell types, namely, endo-, M-, and epicardial cells, is unknown; thus we conducted an exhaustive search strategy for their most likely distribution in the ventricular wall. We varied the distribution of these cell types transmurally and also changed the action potential duration in the apico-basal direction in multiple combinations and simulated the electrical activity of heart and torso. An example of the time course of body surface potential during the QRS complex of the ECG is shown in Fig. 2. Comparing the body surface ECG obtained by various heart models with the body surface potential pattern, we concluded that both transmural and apico-basal gradients in repolarization (APD) contribute to the genesis of normal T-waves in human surface ECG (Fig.3).

Estimating the cell distribution that best reproduces the ECG wave form in this manner constitutes a forward problem approach (as opposed to the inverse problem). Although about 6 hours was required to calculate a single cardiac cycle in this study, with more advanced computation, the approach could be used to identify the most probable tissue properties which match a patients' individual ECG, and could thus become an invaluable tool in tailored medicine.

\section{Sarcomere dynamics}

As mentioned above, most of the contraction models used in heart simulation are based on the cycling cross-bridge model of Huxley (Huxley, 1957), in which myosin heads from the thick filament undergo a series of attachment-detachment cycles with the actin molecules of the thin filament. Incorporation of the $\mathrm{Ca}^{2+}$ dependent regulatory mechanism and/or compliant element into these models now allows accurate simulation of the experimental results obtained under a wide range of skeletal and cardiac muscle conditions, including the twitch contraction and force transient (Hunter et al., 1998; Landesberg and Sideman, 1994; Negroni and Lascano, 2008; Rice et al., 2008). However, to simulate heart activity, these models must capture the anomalously high $\mathrm{Ca}^{2+}$ sensitivity of activation over that of the skeletal muscle. The putative underlying basis of this sensitivity is co-operative interaction between sarcomere components. Among the postulated mechanisms are steric hindrance of tropomyosin by the strongly-bound cross-bridge, and the end-to-end interaction of troponin/tropomyosin units along the thin filament, both of which facilitate the formation of nearby cross-bridge, which then activates the strong response to $\mathrm{Ca}^{2+}$ ions. Obviously, the 
modeling of such mechanisms requires the description of spatially distributed states solved by either partial differential equations (PDE) or Monte Carlo (MC) simulations. The computational cost of these approaches has inspired the development of the mean field model, in which average behavior of the units is formulated by ordinary differential equations (ODEs) with phenomenological parameter tunings (Campbell, 1997; Hunter et al., 1998; Rice et al., 2008). Some of these approaches appear successful but are unable to reproduce the observed rapid force decay during twitch contractions.

Several spatially distributed models have been reported (Daniel et al., 1998; Hussan et al., 2006). In these models, the microanatomical arrangement of sarcomere is preserved and the transition rates of each functional unit are assumed to depend on the state of the neighboring unit and/or the cross-bridge strain. These models yield excellent agreement with experimental observation; however, as stated above, their numerical solution requires computationally expensive MC simulations, thus their application is limited to single sarcomeres or to myofilaments.

Two recent, unique approaches have circumvented the time consuming MC simulations. Rice et al. (Rice et al., 2003) reported an analytical solution of their Ising model but the applicability of this approach seems to be limited to the steady state problem under a simple periodic boundary condition. Campbell et al. (Campbell et al., 2010) adopted a Markov model approach to formulate the transitions between states, which considered every possible combination of neighboring units, rendering their model applicable even to the dynamic twitch contraction. Again, however, the number of units in the model was too few to accurately model sarcomere because the number of required states in Markovian modeling grows explosively with the number of units.

We have developed a novel method for the numerical simulation of spatially-detailed sarcomere dynamics in which MC simulation results are approximated by an ODE (Washio et al., 2011). The framework of the model is schematically presented in Fig. 4. In the model, 36 regulatory units representing the complex of cross-bridge and regulatory proteins are arranged linearly, mimicking the sarcomere structure. Each regulatory unit (indexed as " $i$ ") assumes either of the 4 states defined by the combination of $\mathrm{Ca}^{2+}$ binding (1: bound, 0 : not bound) and cross-bridge formation (P: permissive, N:non-permissive). Transition kinetics are adopted from Rice et al. (Rice et al., 2003), who assumed that transitions between the $\mathrm{N}$ and $\mathrm{P}$ states are governed by the states of their nearest neighbors, i.e., the transition rates are multiplied by the factor $\gamma^{n}$ or $\gamma^{-n}$, where $n$ is the number of P-state nearest-neighbors (inset of Fig. 4). Here, we 
define the vector representing the probability distribution of the $i^{\text {th }}$ unit as follows:

$$
\begin{aligned}
& \boldsymbol{P}_{i \mathrm{i}}={ }^{\mathrm{t}}\left[[0 \mathrm{~N}]_{\mathrm{i}},[1 \mathrm{~N}]_{\mathrm{i}},[1 \mathrm{P}]_{\mathrm{i}},[0 \mathrm{P}]_{\mathrm{i}}\right] \\
& \boldsymbol{P}_{i}^{\xi_{i}, \eta}={ }^{\mathrm{t}}\left[[\xi, 0 \mathrm{~N}, \eta]_{\mathrm{i}},[\xi, 1 \mathrm{~N}, \eta]_{\mathrm{i}},[\xi, 1 \mathrm{P}, \eta]_{\mathrm{i}},[\xi, 0 \mathrm{P}, \eta]_{\mathrm{i}},\right]
\end{aligned}
$$

, where $\xi$ and $\eta$ are the states of the neighboring unit of each side (N or $\mathrm{P})$; thereby $[\xi, \mathrm{x}$, $\eta]$ represents the combinatory probability of the $i^{\text {th }}$ unit being in state $x(0 N, 1 N, 1 P$, or OP) given that its respective neighbors are in states $\xi$ and $\eta$.

The transition kinetics of the $i^{\text {th }}$ unit are described as follows:

$$
\frac{d P_{i}}{d t}=\sum_{\xi_{i}=N_{p} P} A_{i}(\xi, \eta) \cdot P_{i}^{\xi_{i} \eta}
$$

$$
\begin{aligned}
& \boldsymbol{A}_{i}(\xi, \eta) \\
& =\left[\begin{array}{cccc}
-\bar{K}_{\text {on }}[C a]-\gamma^{n} \bar{K}_{m p 0} & K_{\text {off }} & 0 & \gamma^{-n} K_{p m 0} \\
\bar{K}_{o n}[C a] & -K_{o f f}-\gamma^{n} \bar{K}_{m p 1} & \gamma^{-n} K_{p m 1} & 0 \\
0 & \gamma^{n} \bar{K}_{m p 1} & -\gamma^{-n} K_{p m 1}-K^{\prime} & \bar{K}_{\text {off }}^{\prime}[C a] \\
\gamma^{n} \bar{K}_{m p 0} & 0 & K^{\prime}{ }_{\text {off }} & -\gamma^{-n} K_{p m 0}-\bar{K}_{\text {on }}^{\prime}[C a]
\end{array}\right]
\end{aligned}
$$

Because this equation cannot be solved as an ODE without knowing the combinatory probabilities on the right hand side while the variables are the simple state probabilities, we estimated these values based on the MC simulation results.

Briefly, we performed MC simulation by applying dynamically changing $\mathrm{Ca}^{2+}$-transients of various amplitudes. At each time step the number of combinatory states as well as the individual states was counted for each unit to yield the following matrix $\left(W_{i}(\xi, \eta)\right)$

$$
\begin{gathered}
P_{i}^{k \eta} \approx W_{i}(\xi, \eta) \cdot P_{i} \\
W_{i}(\xi, \eta)=\left[\begin{array}{cccc}
W_{i}(\xi, 0 \mathrm{~N}, \eta) & 0 & 0 & 0 \\
0 & W_{i}(\xi, 1 \mathrm{~N}, \eta) & 0 & 0 \\
0 & 0 & W_{i}(\xi, 1 \mathrm{P}, \eta) & 0 \\
0 & 0 & 0 & W_{i}(\xi, 0 \mathrm{P}, \eta)
\end{array}\right],
\end{gathered}
$$


which enables the problem to be solved as an ODE:

$$
\frac{d \boldsymbol{P}_{i}}{d t}=\Sigma_{\xi, \eta=N_{i} P} \boldsymbol{A}_{i}(\xi, \eta) \cdot \boldsymbol{W}_{i}(\xi, \eta) \cdot \boldsymbol{P}_{\tilde{i}}
$$

The results obtained from this ODE agree favorably with the MC results (Fig. 5) The ODE simulation could also reproduce the experimental results under isometric and shortening twitch contractions (Washio et al., 2011). Moreover, the sarcomere dynamics of 1 second duration were solved in just 0.64 seconds using the ODE approximation, while the MC simulation required more than 1000 repeats to obtain reasonable mean values. Although it may be argued that this approach assumes the time-consuming MC simulation as the prerequisite, we have confirmed that the $\boldsymbol{W}$ matrix representing the $\mathrm{Ca}^{2+}$ transients can be applied to other independently obtained experimental transients to accurately model the force data. We consider that once the MC simulation has been conducted for a specific parameter set representing a given sarcomere property (itself driven mainly by properties of functional proteins) established under certain experimental conditions, e.g. $\mathrm{pH}$ or temperature, the $\boldsymbol{W}$ matrix can be used for any other simulations assuming the same cross-bridge property. Furthermore, if we prepare a set of $\boldsymbol{W}$ 's and rate constants for various properties of proteins, for instance, myosins with different degrees of light chain phosphorylation (Davis et al., 2001), we can simulate the dynamics of the heart in which these different myosins are distributed throughout the ventricular wall.

Cooperativity is often defined and evaluated as the shape of the static force-pCa relationship, but it also significantly influences the force transient decay phase during the twitch contraction. In our previous study using the traditional ODE approach, physiological filling of the left ventricle, especially during the rapid filling phase, was not faithfully reproduced (Watanabe et al., 2004b). We will show how our new ODE model can solve this problem in the next section.

\section{Ventricular mechanics}

The relation between the microscopic sarcomere model and the macroscopic ventricular mechanics was examined using the following finite element model of human ventricles. The model was based on our previous human heart model (Okada et al., 2011) and consisted of 77926 tetrahedral elements. We also created tetrahedral meshes in right and left ventricular cavities to calculate their volumes during the cardiac cycle. To each element of this heart model, we implemented the human ventricular myocyte model of electrophysiology (Ten Tusscher et al., 2004; Ten Tusscher and Panfilov, 
2006) and our ODE model of sarcomere dynamics. We also modeled the fiber-sheet structure of myocardium by mapping fiber and sheet angles according to the literature (Costa et al., 1999b). For the sheet angle distribution, their data sets (AF3) at base and apex were adopted and linearly interpolated to cover the whole ventricle.

The constitutive equation for passive myocardium was adopted from Usyk et al. with some modifications (Usyk et al., 2000):

$$
\begin{aligned}
W= & \frac{C\left(e^{Q}-1\right)}{2}+\kappa_{p}(J-1)^{2}+\xi I_{1} \\
Q= & b_{f f} E_{f f}{ }^{2}+b_{s s} E_{s s}{ }^{2}+b_{n n} E_{n n}{ }^{2}+b_{f s}\left({E_{f s}}^{2}+E_{s f}{ }^{2}\right) \\
& \quad+b_{f n}\left(E_{f n}{ }^{2}+E_{n f}{ }^{2}\right)+b_{n s}\left(E_{n s}{ }^{2}+E_{s n}{ }^{2}\right)
\end{aligned}
$$

, where $E_{i j}$ are components of Green's strain tensor defined in the local coordinate system having fiber, sheet and sheet-normal axes, $J$ is the determinant of the deformation gradient tensor, and $I_{l}$ is the first principal reduced invariant. The second term in the first equation is volumetric energy term with $\kappa_{\mathrm{p}}=50 \mathrm{kPa}$ describing the nearly incompressible property and the third term was added to obtain the stability of the model with $\xi=0.07 \mathrm{kPa}$. Other material constants: $\mathrm{C}=0.88 \mathrm{kPa} ; \mathrm{b}_{\mathrm{ff}}=5 ; \mathrm{b}_{\mathrm{ss}}=6 ; \mathrm{b}_{\mathrm{nn}}=3$; $\mathrm{b}_{\mathrm{fs}}=10 ; b_{\mathrm{sn}}=2 ; b_{\mathrm{fs}}=2$ were adjusted to reproduce the physiologically relevant passive pressure-volume relation (Fig. 6B). Contraction was modeled by defining the uniaxial component (fiber direction) of active stress tensor as a function of active force calculated by the sarcomere model. The active stress tensor was added to the passive stress tensor calculated from above constitutive equations to yield the total stress.

For boundary conditions, we fixed the atrio-ventricular ring and coupled ventricular pressure to electrical analog of the circulation (Watanabe et al., 2004b). All the program codes were written in house and computation was performed using IBM Power 6 (JS22) with 128 cores.

Simulated pressure and volume during a single cardiac cycle are shown in Fig. 6A for left (LV: solid line) and right (RV: broken line) ventricles. Due to rapid decline of ventricular pressure attained by the co-operative activation of sarcomere, pressure waveforms became nearly rectangle rather than triangle in shape (upper panel). Time-volume curves (lower panel) can be clearly divided into four phases: 1) isovolumic relaxation phase (horizontal segments in the bottom), 2) early rapid filling phase, during which the majority of filling volume enters the ventricle, 3) diastasis (plateau phase of filling), 4) atrial contraction. All these features characterize the hemodynamics of normal human hearts (Leinihan et al., 1995; Little, 2001). Pressure-volume loops (Fig. 6B) also show normal cardiac function (LV Ejection 
fraction $=59 \%$, RV ejection fraction $=50 \%)$ (Gentzler II et al., 1974). In particular, the bottom part of the loop follows the exponential diastolic pressure-volume relation as reported in the literature for normal human hearts (Little, 2001). This physiological diastolic pressure-volume relation is an indication of complete relaxation and shows clear contrast to those reported in other electromechanical simulation studies (Gurev et al., 2010; Kerckhoffs et al., 2006). To further examine the effect of cooperativity on ventricular mechanics, we eliminated the cooperative effect from sarcomere model (setting $\gamma=1$ in Fig. 4), and repeated the simulation. As shown in Fig. 6B, this intervention severely impaired both systolic and diastolic functions so that pressure-volume loops were compressed and shifted leftward (LV: red solid line, RV: red broken line). Because it was hard to gain insights from these distorted pressure-volume loops, we compared time-volume curves of LV with and without cooperativity by normalizing them relative to their maximum and minimum values (Fig 6C). It is clearly seen that, without cooperativity (red line), early filling became slow and the fraction of filling volume during this period was decreased. These findings are considered as indications of diastolic dysfunction in clinical settings (Leinihan et al., 1995). On the other hand, ejection was accelerated by the elimination of cooperativity because cooperative mechanism of activation rather inhibits the force development at very low calcium concentration while enhancing it as concentration increases.

In addition to the sarcomere dynamics, many microscopic structural components are implicated in the ventricular mechanics. Among these, fiber orientation has been the focus of intensive studies and those studies unanimously reported a quasi-linear gradient of fiber angle across the ventricular wall (Ashikaga et al., 2004; Costa et al., 1999a; Omens et al., 1991; Streeter Jr et al., 1969). Simulation studies demonstrated that the optimal fiber distribution for homogeneous fiber strain during ejection (Rijcken et al., 1999) and for maximizing the external work is close to such anatomical measurements. Similarly, changes in laminar sheet angle during contraction have been reported (Ashikaga et al., 2005; Costa et al., 1999a) and the simulation study has been attempted to find that incorporation of orthotropic model with sheet structure to the model yields better agreement with the measured strain distribution (Usyk et al., 2000). In this simulation, we also introduced the orthotropic passive property to reproduce the physiological diastolic pressure-volume relation, but further analyses are required to reveal the functional impact of sheet structure in more detail.

\section{Hemodynamics}

Because the essential function of the heart is to pump blood, fluid dynamics is an 
indispensable part of whole heart modeling and also an important issue in clinical cardiology. To date, however, only a few whole heart models have considered this aspect due to the complexity and the difficulty of the problem. To establish dynamic equilibrium, the kinematic energy of the blood flow is transferred from the contracting myocardial wall; such active contraction causes shape changes in the wall. In this sense, hemodynamics problems involve fluid structure interaction and large deformation analyses. The strategies adopted thus far include the immersed boundary method or moving boundary method (Kovacs et al., 2001; Nakamura et al., 2003) but the important clinical questions, such as the relation between the flow velocity distribution and the tissue property, remain unanswered. We have approached this problem using the Arbitrary Lagrangian Eulerian (ALE) finite element method with automatic mesh-updating developed in our laboratory (Zhang and Hisada, 2001), a form of interface-tracking. Briefly, the ALE forms of the Navier-Stokes equations are discretized in the fluid domain. On the structural domain, the equilibrium equations are formulated solely in Lagrangian form and discretized.

The fluid and structural domains are coupled by equating the displacement, velocity and acceleration on the interface through the element assemblage process. The system equations in the strong coupling method were solved simultaneously using a monolithic matrix.

Taking advantage of the computational accuracy and stability of this method, we have simulated the flow dynamics in the left ventricle during the cardiac cycle. In this simulation, pre- and after-loads to the left ventricle were modeled by the electric analog circuits of circulation with a contracting left atrium represented by the time-varying elastance (Alexander et al., 1987) (Fig.7A). Propagation of excitation was formulated by the Fitz-Hugh-Nagumo model and the sarcomere dynamics were modeled by the 4-state Markov model. As observed in the human heart (Kilner et al., 2000), the inflow to the ventricle changes its direction to form a vortex and is finally squeezed out from the ventricle (Fig. 7B). As a result, only a fraction of the incoming blood during each diastole is ejected during the next systole. Our analysis revealed that blood entering during the first beat is completely cleared from the ventricle after around 9 beats at low heart rate (Watanabe et al., 2008). The functional significance of vortex formation is not clear but, at least in our analysis, the momentum of the blood heading towards the outflow tract was negligible and the kinetic energy of the ejected blood came principally from the work performed by the contraction of left ventricular myocardium. We have also found differences in the intra-ventricular flow patterns between the heart model with myocardial infarction and that of the normal heart during systole and diastole 
(Watanabe et al., 2004a). Furthermore, diastolic dysfunction (introduced by changing the parameter in the sarcoplasmic reticulum $\mathrm{Ca}^{2+}$ pump) impaired the diastolic rapid filling flow (unpublished data). These results highlight the importance of fluid-structure interaction analysis which could establish links between the subcellular molecular mechanics and the clinically observed hemodynamic parameters.

Another clinically important issue relevant to heart fluid dynamics is the motion of valves, which cause dramatic organ deformations in instances of high blood flow velocity. Previous aortic valve studies using either the commercially available software package (Nicosia et al., 2003) or the fictitious domain method (De Hart et al., 2003), were unable to analyze this problem at a physiologically relevant Reynolds number due to numerical instability. By employing the ALE finite element method with an automatic remeshing and reconnecting algorithm to correct for the large movement of the fluid-structure interface, we have been able to simulate the motion of aortic valve under a physiological Reynolds number ( 3000, flow velocity $\fallingdotseq 1 \mathrm{~m} / \mathrm{sec}$ ) (Katayama et al., 2008). In this analysis, anisotropic aortic valve leaflets were coupled to the short segment of the aorta. The sinus of Valsalva was found to facilitate the gradual but smooth closure of the valve by channeling blood into a vortex on the distal side of each leaflet during the early phase of ejection, thereby reducing stresses on the leaflet (Fig. $7 \mathrm{C})$.

\section{Conclusion and Perspective}

The need to integrate the vast amount of knowledge being gained at the molecular and cellular levels by comprehensive modeling is becoming more urgent, as understanding the function of the heart in depth will improve the accuracy and safety of clinical cardiology practices. The development and advancement of various novel diagnostic modalities requires that heart simulators be not only multi-scale but also multi-physics, in order to accurately compare with and examine multi-faceted clinical data. We have reviewed the necessary components and methodologies for the realization of such an electro-mechano-hemodynamic heart model. Assembly of these components to construct a comprehensive heart model is computationally costly but we have successfully developed a prototype model (Fig. 8 http://www.sml.k.u-tokyo.ac.jp/). With further validation and sophistication, and inclusion of missing components and details such as metabolism (Cortassa et al., 2006 ; Hatano et al., 2011), we hope that our heart simulator will become a valuable tool in clinical cardiology practice. 
The starting point for the modeling process is an issue of ongoing debate (Noble, 2006). Due to present limitations in computational power, the cellular-level starting point of virtually all heart models is a lumped parameter system. Although apparently driven by the ionic current controlled by ion channels, pumps, or transporters, the functions of intracellular molecules are described in terms of per cell or per unit area of cell membrane, while ignoring their subcellular distributions (hence the term "lumped parameter model"). This "middle out" approach (Noble, 2006)has been successful to date and is also practical. However, if the starting point could be refined to a lower level to accommodate subcellular structure, the capability of the model to probe the mechanisms of intricate biological systems would be further intensified.

The capacity of high performance computers is increasing rapidly. In 2011, the K-computer developed by RIKEN and Fujitsu, comprising 864 computer racks equipped with a total of 88,128 CPUs, has achieved the world's highest LINPACK benchmark performance (10.51 petaflops). Even at this level of performance, a whole heart model based on the dynamics of each molecule in the myocyte could not be accomplished. However, the periodic nature of myocardial tissue may justify the computational technique of homogenization, by which a virtual heart composed of myocytes with subcellular structures, at least in part, may be maintained in the computer (Okada et al., 2010). Of course, this will be an enormous computational task with more than 10 trillion degrees of freedom, but we are aiming at its development.

\section{Acknowledgement}

This work was supported by the Japan Science and Technology Agency under grant Collaborative Development of Innovative Seeds-Practicability Verification Stage, and Japan Society for the Promotion of Science (JSPS) through its "Funding Program for World-Leading Innovative R\&D on Science and Technology (FIRST Program)".

\section{Disclosure}

The University of Tokyo/Graduate School of Frontier Sciences receives grant support from Fujitsu Ltd. 


\section{Fig. 4 Spatially-detailed model of sarcomere dynamics}

Regulatory units assuming the complex of cross-bridge on the thick filament and regulatory proteins on the thin filament are arranged linearly. Each regulatory unit (indexed as " $\mathrm{i}$ ") takes either of the 4 states defined by the combination of $\mathrm{Ca}^{2+}$ binding (1: bound, 0: not bound) and cross-bridge formation (P: permissive, N: non-permissive). Transition kinetics are adopted from Rice et al. (Rice et al., 2003). The rate constants of transition between the states $\left(\bar{K}_{o n}, K_{\text {off }}, \bar{K}_{o n}^{\prime}, K_{o f f}^{\prime}, K_{p m 1}, \bar{K}_{p m 1}, K_{p m 0}, \bar{K}_{p m 0}\right)$ are modified by multiplication with either $\left[\mathrm{Ca}^{2+}\right]$ or factors $\tilde{a}^{n}$ or $\tilde{a}^{-n}$ representing the states of the nearest neighbors.

\section{Fig. 5 Comparison between $\mathrm{MC}$ and $\mathrm{ODE}$ results}

$\mathrm{MC}$ and ODE simulations were performed under isometric condition. Twitch forces ([P] values) in response to the two representative $\mathrm{Ca}^{2+}$ transients (top row) were calculated using MC (red circles) and ODE (solid lines) simulations at various sarcomere lengths $(\mathrm{SL}=1.5,1.8,2.3 \mu \mathrm{m})\left(2^{\text {nd }}\right.$ to $4^{\text {th }}$ rows $)$. 


\section{Fig. 6 Ventricular mechanics}

A: Pressure (upper panel) and volume (lower panel) waveforms of left (solid line) and right (broken line) ventricles. B: Pressure-volume loops of left (solid line) and right (broken line) ventricles with (black) or without (red) cooperativity. C: Time-volume curves of the left ventricle normalized by its maximum and minimum values with (black) and without (red) cooperativity.

\section{Fig. 7 Fluid structure interaction analysis of the left ventricular hemodynamics}

A: Diagram of macroscopic model. FEM meshed for solid (ventricular wall) and fluid (blood) elements are shown with their electrical analogs of afterload (systemic arterial tree) and preload (pulmonary circulation with active LA). PV, pulmonary source pressure; RV, source resistance; $\mathrm{CP}$, pulmonary venous capacitance; $\mathrm{RP}$, pulmonary resistance; LP, pulmonary inertance; RAV, atrioventricular resistance; LAV, atrioventricular inertance; FRL, pulmonary venous flow; FMi, blood flow through the mitral valve; qP and qLA, volumes of the pulmonary venous and LA. (From Fig. 2 of Watanabe et al. (Watanabe et al., 2004b) with permission)

B: Time-lapse images of LV contraction and intraventricular blood flow. Numbers in each plate indicate the time following the onset of activation in seconds. Activation level (relative force) is shown in color coding. Velocity of blood is represented by the length of the arrows (From Fig. 4 of Watanabe et al. (Watanabe et al., 2004b) with permission)

C: Stress in the leaflet and deformation of aortic valve. Close-up view of the leaflet in the late phase of ejection is shown with stress values in color coding (Modified from Fig. 5A of Katayama et al. (Katayama et al., 2008) with permission)

\section{Fig. 8 Multi-scale, multi-physics heart simulation}

The heart model on the left shows the propagation of excitation signals and the model on the right shows the blood flow in the heart chamber. The relevance of the indicated components is discussed in the text. Videos can be seen at http://www.sml.k.u-tokyo.ac.jp/. 


\section{References}

Alexander, J., Sunagawa, K., Chang, N. and Sagawa, K., 1987. Instantaneous pressure-volume relation of the ejecting canine left atrium, Circ Res. 61, 209-219.

Ashikaga, H., Covell, J.W. and Omens, J.H., 2005. Diastolic dysfunction in volume-overload hypertrophy is associated with abnormal shearing of myolaminar sheets, Am J Physiol. 288, H2603-H2610.

Ashikaga, H., Criscione, J.C., Omens, J.H., Covell, J.W. and Ingels, N.B., 2004. Transmural left ventricular mechanics underlying torsional recoil during relaxation, Am J Physiol. 286, H640-H647.

Bazil, J.N., Buzzard, G.T. and Rundell, A.E., 2010. Modeling mitochondrial bioenergetics with integrated volume dynamics, PLoS Comp Biol 6, e1000632.

Beeler, G.W. and Reuter, H., 1977. Reconstruction of the action potential of ventricular myocardial fibers, J Physiol. 268, 177-210.

Beyar, R. and Sideman, S., 1984. A computer study of the left ventricular performance based on fiber structure, sarcomere dynamics, and transmural electrical propagation velocity, Circ Res. 55, 358-75.

Campbell, K., 1997. Rate constant of muscle force redevelopment reflects cooperative activation as well as cross-bridge kinetics, Biophys J. 72, 254-262.

Campbell, S.G., Lionetti, F.V., Campbell, K.S. and McCulloch, A.D., 2010. Coupling of adjacent tropomyosins enhances cross-bridge-mediated cooperative activation in a Markov model of the cardiac thin filament, Biophys J. 98, 2254-2264.

Cheng, Y., Yu, Z., Hoshijima, M., Holst, M.J., McCulloch, A.D., McCammon, J.A. and Michailova, A.P., 2010. Numerical analysis of $\mathrm{Ca}^{2+}$ signaling in rat ventricular myocytes with realistic transverse-axial tubular geometry and inhibited sarcoplasmic reticulum, PLoS Comput Biol. 6, e1000972.

Cortassa, S., Aon, M.A., Marban, E., Winslow, R.L. and O'Rourke, B., 2003 An integrated model of cardiac mitochondrial energy metabolism and calcium dynamics, Biophys J. 84, 2734-2755.

Cortassa, S., Aon, M.A., O'Rourke, B., Jacques, R., Tseng, H.-J., Marban, E. and 
Winslow, R.L., 2006 A computational model Integrating electrophysiology, contraction, and mitochondrial bioenergetics in the ventricular myocyte, Biophys J. 91 1564-1589.

Costa, K.D., Takayama, Y., McCulloch, A.D. and Covell, J.W., 1999a. Laminar fiber architecture and three-dimensional systolic mechanics in canine ventricular myocardium, Am J Physiol 276, 595-607.

Costa, K.D., Takayama, Y., McCulloch, A.D. and Covell, J.W., 1999b. Laminar fiber architecture and three-dimensional systolic mechanics in canine ventricular myocardium, Am J Physiol. 276, H595-H607.

Daniel, T.L., Trimble, A.C. and P.B., C., 1998. Compliant realignment of binding sites in muscle: transient behavior and mechanical tuning, Biophys J. 74, 1611-1621.

Davis, J.S., Hassanzadeh, S., Winitsky, S., Lin, H., Satorius, C., Vemuri, R., Aletras, A.H., Wen, H. and Epstein, N.D., 2001. The overall pattern of cardiac contraction depends on a spatial gradient of myosin regulatory light chain phosphorylation, Cell. 107, 631-641.

De Hart, J., Peters, G.W., Schreurs, P.J. and Baaijens, F.P., 2003. A three-dimensional computational analysis of fluid-structure interaction in the aortic valve, $\mathrm{J}$ Biomech. 36, 103-112.

Dupont, G., Pontes, J. and Goldbeter, A., 1996. Modeling spiral $\mathrm{Ca}^{2+}$ waves in single cardiac cells: role of the spiral heterogeneity created by the nucleus, Am J Physiol 271, C1390-C1399.

Durrer, D., van Dam, R.T., Freud, G.E., Janse, M.J., Meijler, F.L. and Arzbaecher, R.C., 1970. Total excitation of the isolated human heart, Circulation 41, 899-912.

Farina, D., Jiang, Y. and Do“ssel, O., 2009. Acceleration of FEM-based transfer matrix computation for forward and inverse problems of electrocardiography, Med Biol Eng Comput 47, 1229-1236.

Fischer, G., Tilg, B., Modre, R., Huiskamp, G.J.M., Fetzer, J., Rucker, W. and Wach, P., 2000. A bidomain model based BEM-FEM coupling formulation for anisotropic cardiac tissue, Ann Biomed Eng. 28, 1229-1243.

Gentzler II, R.D., Briselli, M.F. and Gault, J.H., 1974. Angiographic estimation of right ventricular volume in man, Circulation 50, 324-330.

Gurev, V., Constantino, J., Rice, J.J. and Trayanova, N.A., 2010. Distribution of electromechanical delay in the heart: Insights from a three-dimensional electomechanical model, Biophys J. 99, 745-754.

Hatano, A., Okada, J., Washio, T., Hisada, T. and Sugiura, S., 2011. A three-dimensional simulation model of cardiomyocyte integrating excitation-contraction coupling 
and metabolism, Biophys J. 101, 2601-2610.

Hunter, P.J. and Borg, T.K., 2003. Integration from proteins to organs: the Physiome Project, Nature Rev. 4, 237-243.

Hunter, P.J., McCulloch, A.D. and ter Keurs, H.E.D.J., 1998. Modeling the mechanical properties of cardiac muscle, Prog Biophys Mol Biol. 69, 289-331.

Hussan, J., de Tombe, P.P. and Rice, J.J., 2006. A spatially detailed myofilament model as a basis for large-scale biological simulations, IBM J RES \& DEV. 50, 583-600.

Huxley, A.F., 1957. Muscle structure and theories of contraction, Prog Biophys Biophys Chem. 7, 255-318.

Izu, L.T., Wier, W.G. and Balke, C.W., 2001. Evolution of cardiac calcium waves from stochastic calcium sparks, Biophys J 80, 103-120,

Katayama, S., Umetani, N., Sugiura, S. and Hisada, T., 2008. The sinus of Valsalva relieves abnormal stress on aortic valve leaflets by facilitating smooth closure, $\mathrm{J}$ Thorac Cardiovasc Surg. 136 1529-1535.

Keizer, J. and Smith, G.D., 1998. Spark-to-wave transition: saltatory transmission of calcium waves in cardiac myocytes, Biophys Chem 72, 87-100.

Keller, D.U.J., Jarrousse, O., Fritz, T., Ley, S., Dossel, O. and Seemann, G., 2011. Impact of physiological ventricular deformation on the morphology of the T-wave: a hybrid, static-dynamic approach, IEEE Transactions on Biomedical Engineering. 58, 2109-19.

Keller, D.U.J., Weber, F.M., Seemann, G. and Dossel, O., 2010. Ranking the Influence of Tissue Conductivities on Forward-Calculated ECGs, IEEE Trans Biomed Eng. 57.

Kerckhoffs, R.C.P., Neal, M.L., Gu, Q., bassingthwaighte, J.B., Omens, J.H. and McCulloch, A.D., 2006. Coupling of a 3D finite element model of cardiac ventricular mechanics to lumped systems models of the systemic and pulmonic circulation, Ann Biomed Eng. 35, 1-18.

Kilner, P.J., Yang, G.Z., Wilkes, A.J., Mohiaddin, R.H., Firmin, D.N. and Yacoub, M.H., 2000. Asymmetric redirection of flow through the heart, Nature 404, 759-761.

Kovacs, S., McQueen, D.M. and Peskin, C.S., 2001. Modelling cardiac fluid dynamics and diastolic function, Phil. Trans. R. Soc. A. 359, 1299-1314.

Landesberg, A. and Sideman, S., 1994. Mechanical regulation of cardiac muscle by coupling calcium kinetics with cross-bridge cycling, Am J Physiol. 267, H779-H795.

Lee, J., Niederer, S., Nordsletten, D., Le Grice, I., Smaill, B., Kay, D. and Smith, N., 
2009. Coupling contraction, excitation, ventricular and coronary blood flow across scale and physics in the heart, Phil. Trans. R. Soc. A. 367, 2311-31.

Leinihan, D.J., Gerson, M.C., Hoit, B.D. and Walsh, R.A., 1995. Mechanisms, diagnosis, and treatment of diastolic heart failure, Am Heart J. 130, 153-166.

Little, W.C., 2001. Assessment of normal and abnormal cardiac function, in: Braunwald, E., Zipes, D.P. and Libby, P. (Eds.), Heart Disease. W.B. Saunders, Philadelphia.

Lu, S., Michailova, A., Saucerman, J., Cheng, Y., Yu, Z., Kaiser, T., Li, W., Bank, R., Holst, M., McCammon, J., Hayashi, T., Hoshijima, M., Arzberger, P. and McCulloch, A., 2009. Multiscale modeling in rodent ventricular myocytes.[Erratum appears in IEEE Eng Med Biol Mag. 2009 May-Jun;28(3):65], IEEE Engineering in Medicine \& Biology Magazine. 28, 46-57.

Luo, C. and Rudy, Y., 1994 A dynamic model of the cardiac ventricular action potential - simulatons of ionic currents and concentration changes Circ Res. 74, 1071-1097.

Maack, C., Cortassa, S., Aon, M.A., Liu, T. and O'Rourke, B., 2006. Elevated cytosolic $\mathrm{Na}^{+}$decreases mitochondrial $\mathrm{Ca}^{2+}$ uptake during excitation-contraction coupling and

impairs energetic adaptation in cardiac myocytes, Circ Res. 99, 172-182.

Nakamura, M., Wada, S., Mikami, T., Kitabatake, A. and Karino, T., 2003. Computational study on the evolution of an intraventricular vortical flow during early diastole for the interpretation of color M-mode Doppler echocardiograms, Biomech Model Mechanobiol. 2, 59-72.

Negroni, J.A. and Lascano, E.C., 1996. A cardiac muscle model relating sarcomere dynamics to calcium kinetics, J Mol Cell Cardiol. 28, 915-929.

Negroni, J.A. and Lascano, E.C., 2008. Simulation of steady state and transient cardiac muscle response experiments with a Huxley-based contraction model, J Mol Cell Cardiol. 45, 300-312.

Nicosia, M.A., Cochran, R.P., Einstein, D.R., Rutland, C.J. and Kunzelman, K.S., 2003. A coupled fluid-structure finite element model of the aortic valve root, J Heart Valve Dis. 12, 781-789.

Noble, D., 1960. Cardiac action and pacemaker potentials based on the Hodgkin-Huxley equations, Nature. 188, 495-7.

Noble, D., 2002. Modelling the heart: from genes to cells to the whole organ, Science. 295, 1678-1682.

Noble, D., 2006. 6. The orchestra: Organs and systems of the body, The music of life. 
Oxford University Press, New York, pp. 74-87.

Okada, J., Sugiura, S., Nishimura, S. and Hisada, T., 2005. Three-dimensional simulation of calcium waves and contraction in cardiomyocytes using the finite element method, Am J Physiol. 288, C510-C522.

Okada, J., Washio, T. and Hisada, T., 2010. Study of efficient homogenization algorithms for nonlinear problems Approximation of a homogenized tangent stiffness to reduce computational cost, Comput Mech 46, 247-258.

Okada, J., Washio, T., Maehara, A., Momomura, S., Sugiura, S. and Hisada, T., 2011. Transmural and apicobasal gradients in repolarization contribute to T-wave genesis in human surface ECG, Am J Physiol. 301, H200-208.

Omens, J.H., May, K.D. and McCulloch, A.D., 1991. Transmural distribution of three-deimensional strain in the isolated arrested canine left ventricle, Am J Physiol. 261, H918-928.

Potse, M., Dubé, B., Richer, J., Vinet, A. and Gulrajani, R.M., 2006. A Comparison of Monodomain and Bidomain Reaction-Diffusion Models for Action Potential Propagation in the Human Heart, IEEE Trans Biomed Eng 53, 2425-2435.

Ramanathan, C., Ghanem, R.N., Jia, P., Ryu, K. and Rudy, Y., 2004. Noninvasive electrocardiographic imaging for cardiac electrophysiology and arrhythmia, Nature Medicine. 10, 422-8.

Rice, J.J., Stolovitzky, G., Tu, Y. and de Tombe, P.P., 2003. Ising model of cardiac thin filament activation with nearest-neighbor cooperative interactions, Biophys J. 84, 897-909.

Rice, J.J., Wang, F., Bers, D.M. and de Tombe, P.P., 2008. Approximate model of cooperative activation and crossbridge cycling in cardiac muscle using ordinary differential equations., Biophys J 95, 2368-2390.

Rijcken, J., Bovendeerd, P.H.M., Schoofs, A.J.G., van Campen, D.H. and Arts, T., 1999. Optimization of cardiac fiber orientation for homogeneous fiber strain during ejection, Ann Biomed Eng. 27, 289-297.

Shannon, T.R., Wang, F., Puglisi, J., Weber, C. and Bers, D.M., 2004. A mathematical treatment of integrated $\mathrm{Ca}$ dynamics within the ventricular myocyte, Biophys $\mathrm{J}$. 87, 3351-3371.

Silva, J.R., Pan, H., Wu, D., Nekouzadeh, A., Decker, K.F., Cui, J., Baker, N.A., Sept, D. and Rudy, Y., 2009. A multiscale model linking ion-channel molecular dynamics and electrostatics to the cardiac action potential, Proc Natl Acad Sci USA. 106, 11102-11106.

Stewart, P., Aslanidi, O.V., Noble, D., Noble, P.J., Boyett, M.R. and Zhang, H., 2009 
Mathematical models of the electrical action potential of Purkinje fibre cells, Phil. Trans. R. Soc. A 367, 2225-2255.

Streeter Jr, D.D., Spotnitz, H.M., Patel, D.P., Ross Jr, J.R. and Sonnenblick, E.D., 1969. Fiber orientation in the canine left venticle during diastole and systole, Circ Res. 24, 339-347.

Sunagawa, K. and Sagawa, K., 1982. Models of ventricular contraction based on time-varying elastance, Crit Rev Biomed Eng. 7, 193-228.

Ten Tusscher, K.H.W.J., Noble, D., Noble, P.J. and Panfilov, A.V., 2004. A model for human ventricular tissue, Am J Physiol. 286, H1573-H1589.

Ten Tusscher, K.H.W.J. and Panfilov, A.V., 2006. Alternans and spoiral breakup in a human ventricular tissue model, Am J Physiol. 291, H1088-H1100.

Trayanova, N.A., 2011. Whole-heart modeling Applications to cardiac electrophysiology and electromechanics, Circ Res. 108, 113-128.

Usyk, T.P., Mazhari, R. and McCulloch, A.D., 2000. Effect of laminar orthotropic myofiber architecture of regional stress strai in the canine left ventricle, $\mathrm{J}$ Elasticity. 61, 143-164.

Vigmond, E., Vadakkumpadan, F., Gurev, V., Arevalo, H., Deo, M., G., P. and Trayanova, N., 2009. Towards predictive modelling of the electrophysiology of the heart, Exp Physiol. 94, 563-577.

Washio, T., Okada, J. and Hisada, T., 2010. A parallel multilevel technique for solving the bidomain equation on a human heart with Purkinje fibers and a torso model, SIAM Review 52, 717-743.

Washio, T., Okada, J., Sugiura, S. and Hisada, T., 2011. Approximation for cooperative interactions of a spatially-detailed cardiac sarcomere model, Cell Mol Bioeng.

Watanabe, H., Sugano, T., Sugiura, S. and Hisada, T., 2004a. Finite element analysis of ventricular wall motion and intra-ventricular blood flow in heart with myocardial infarction, JSME Internat J C. 47, 1019-1026.

Watanabe, H., Sugiura, S. and Hisada, T., 2008. The looped heart does not save energy by maintaining the momentum of blood flowing in the ventricle, Am J Physiol. 294, H2191-H2196.

Watanabe, H., Sugiura, S., Kafuku, H. and Hisada, T., 2004b. Multiphysics simulation of left ventricular filling dynamics using fluid-structure interaction finite element method, Biophys J. 87, 2074-2085.

Winslow, R.L., Greenstein, J.L., Tomaselli, G.F. and O'Rouke, B., 2001. Computational models of the failing myocyte: relating altered gene expression to cellular function, Phil. Trans. R. Soc. A. 359, 1187-1200. 
Xue, J., Gao, W., Chen, Y. and Han, X., 2009. Identidy drug-induced T wave morphology changes by a cell-to-electrocardiogram model and validation with clinical trial data, J Electrocardiol. 42, 534-542.

Zhang, Q. and Hisada, T., 2001. Analysis of fluid-structure interaction problem with structural buckling and large domain change by ALE finite element method, Comput Methods Appl Mech Engrg. 190, 6341-6357. 
Fig.1

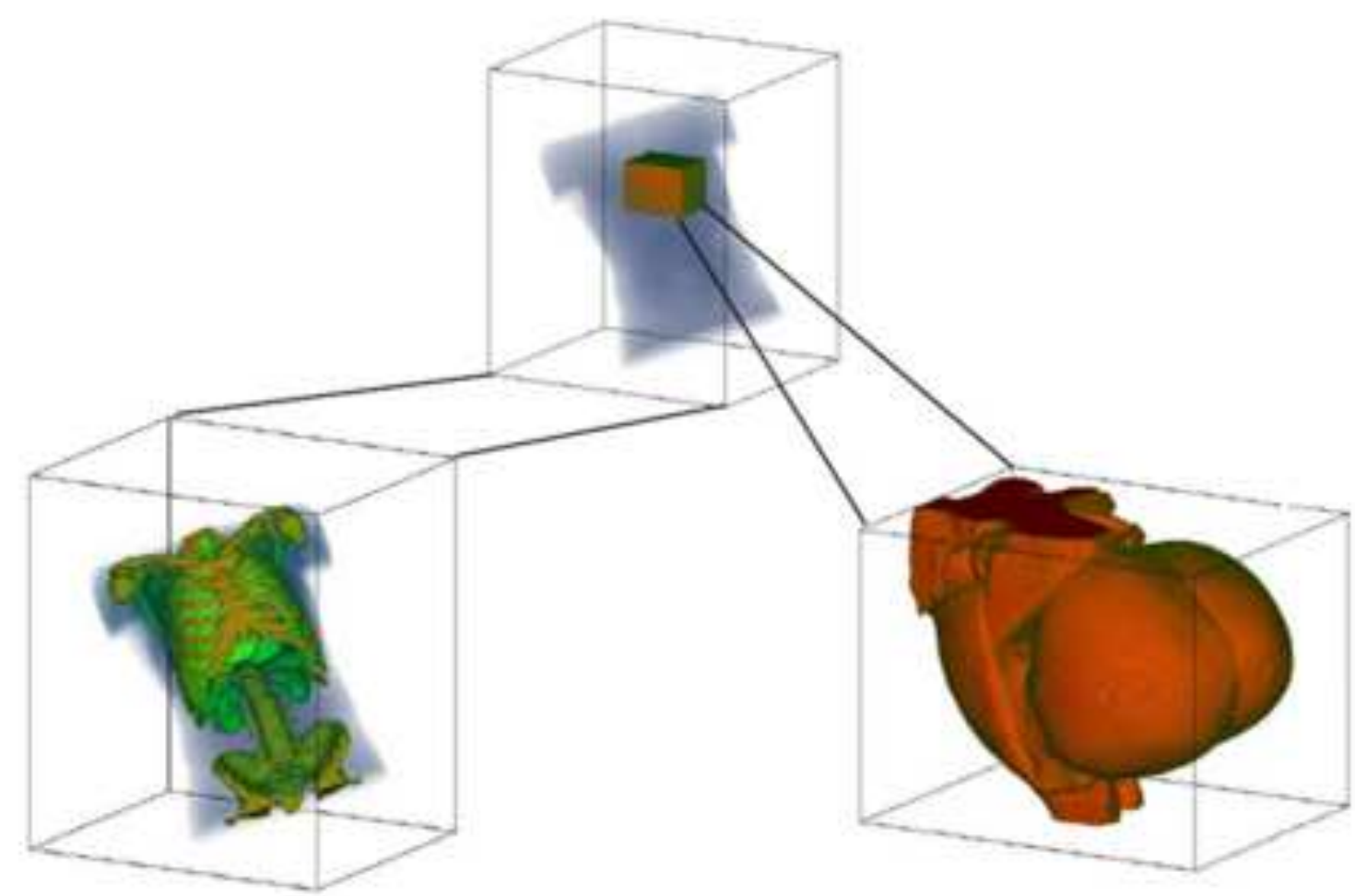

Torso mesh $(1.6 \mathrm{~mm})$

Heart mesh $(0.2 \mathrm{~mm})$ 
Fig.2
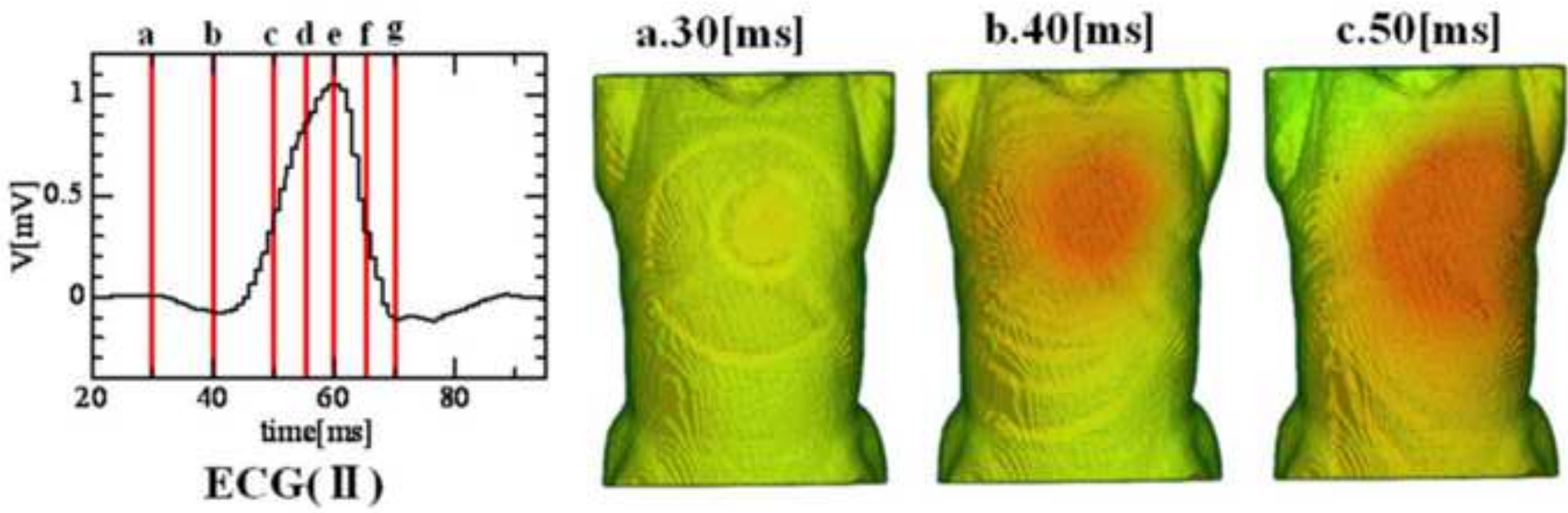

d. $55[\mathrm{~ms}]$

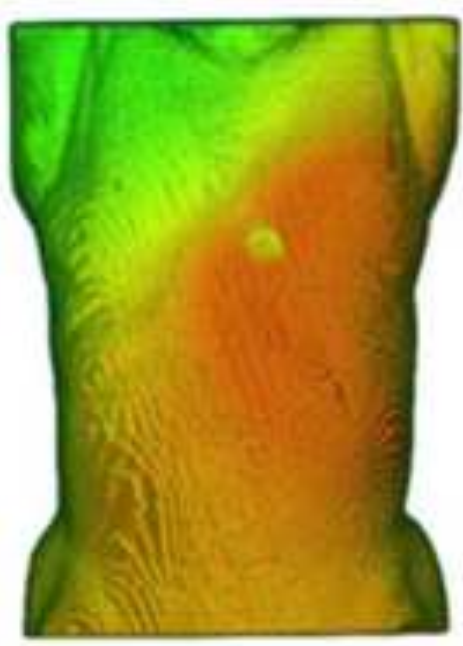

e. $60[\mathrm{~ms}]$

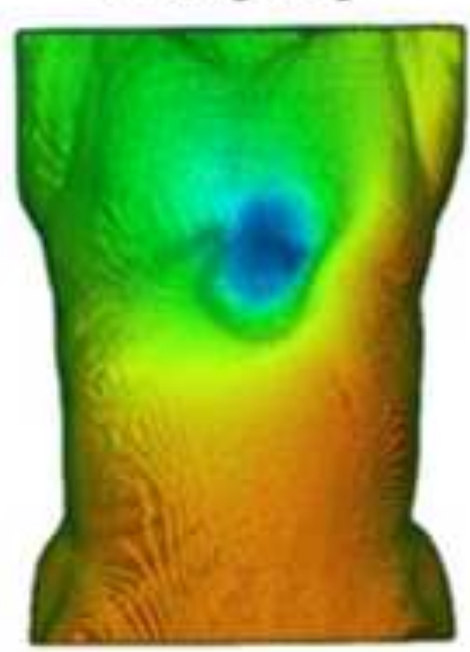

f. $65[\mathrm{~ms}]$

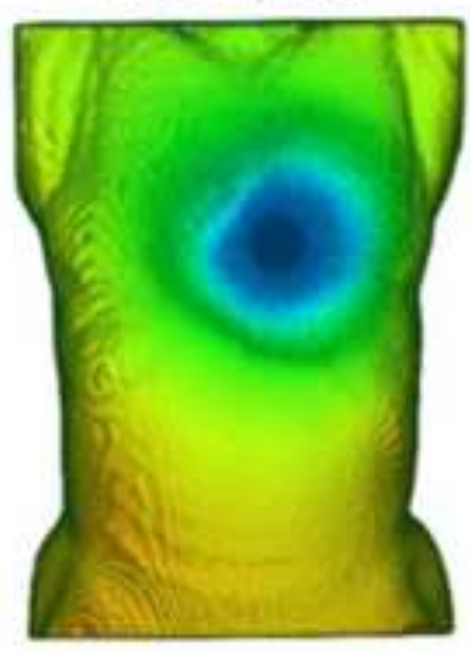

g. $70[\mathrm{~ms}]$

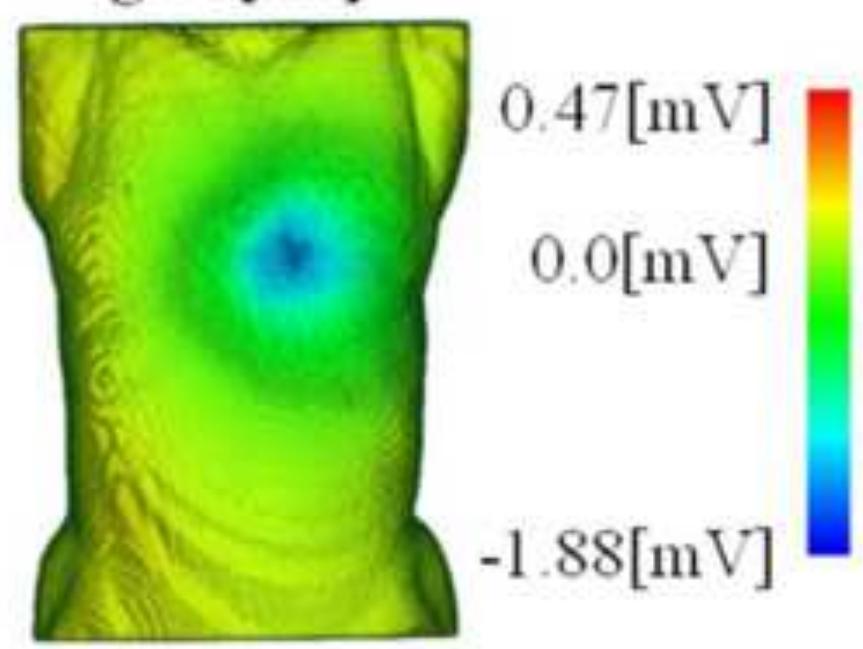




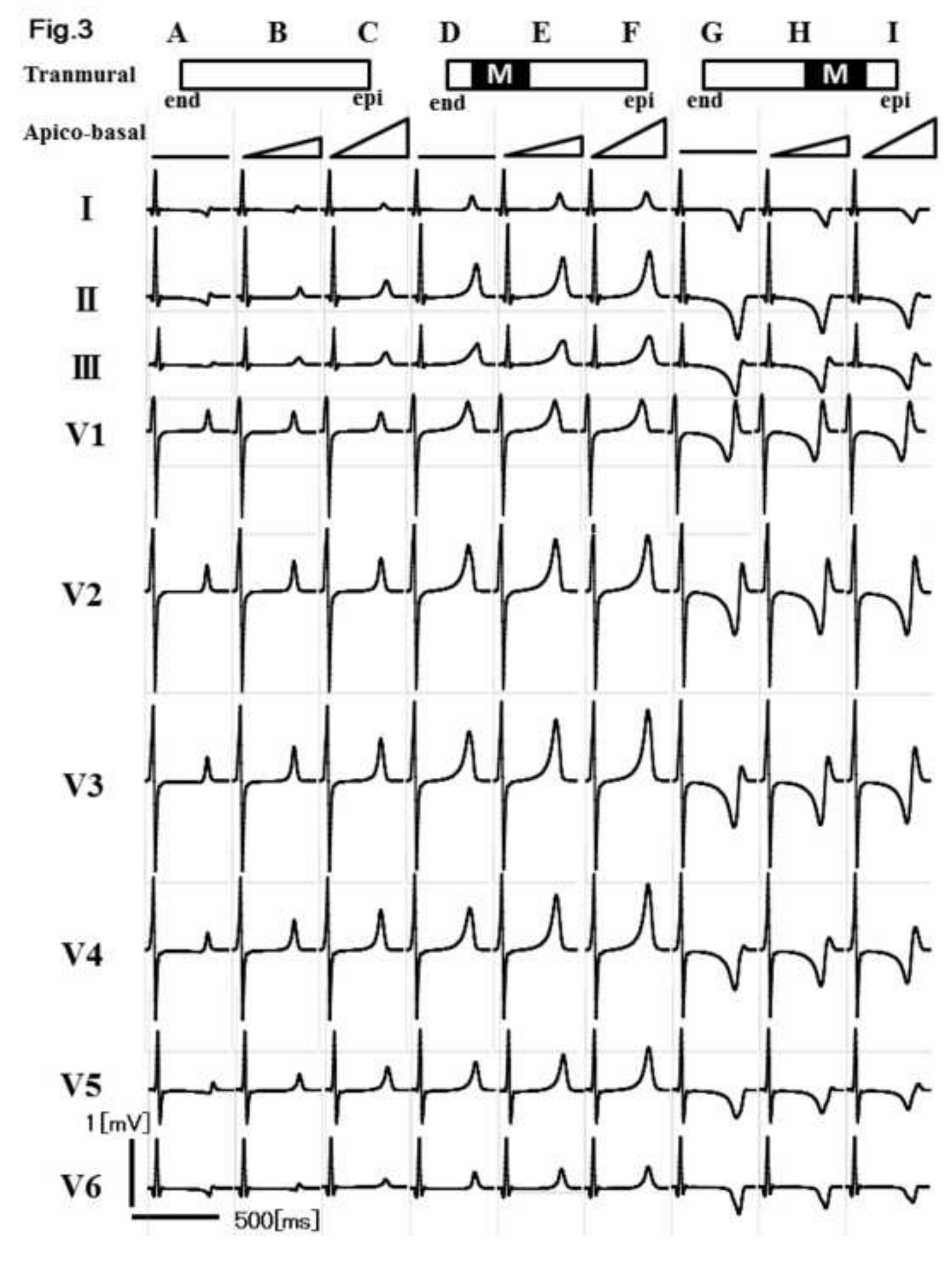




Fig.4



Thin filament

Thick filament 
Fig.5
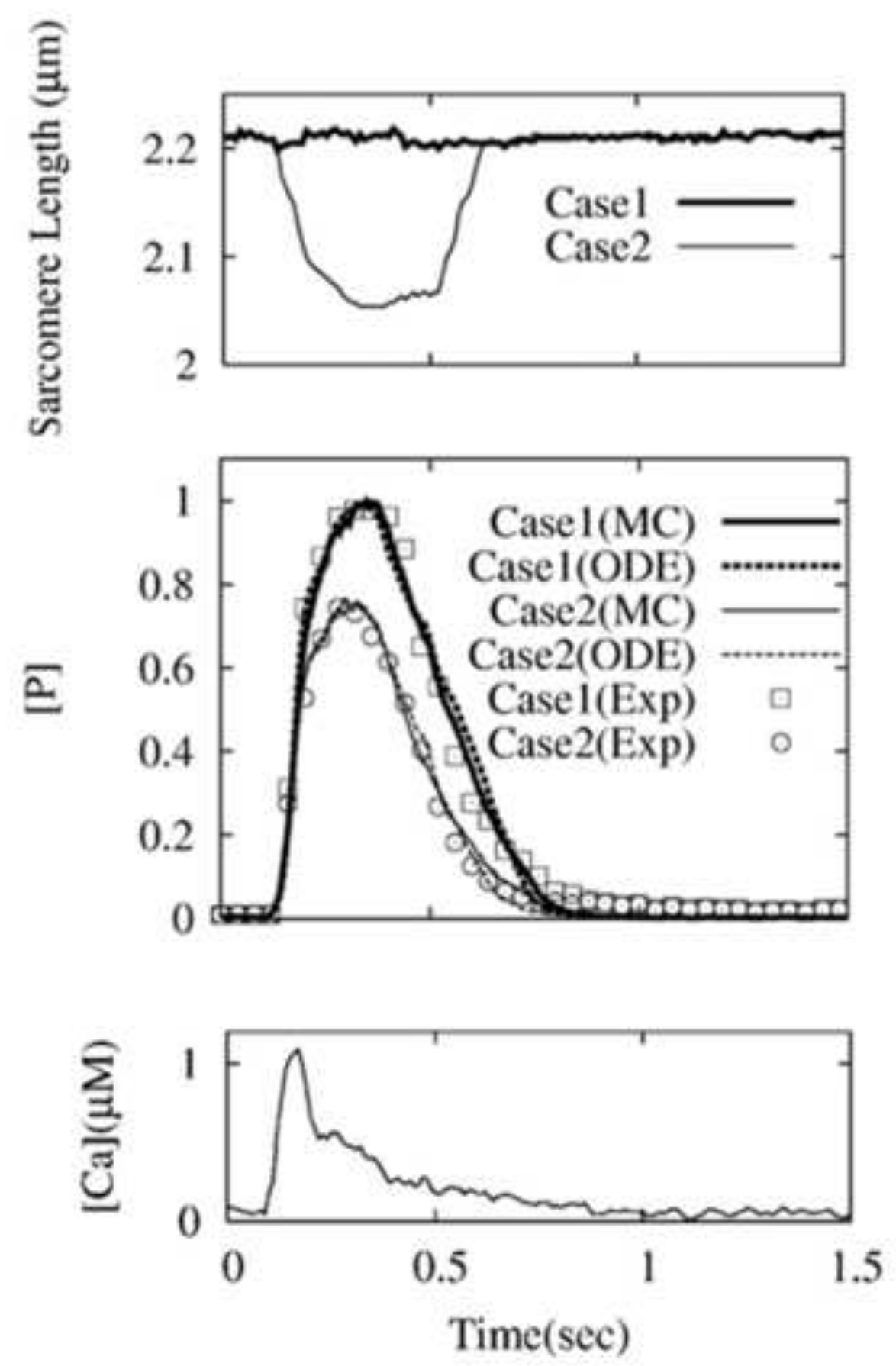
A
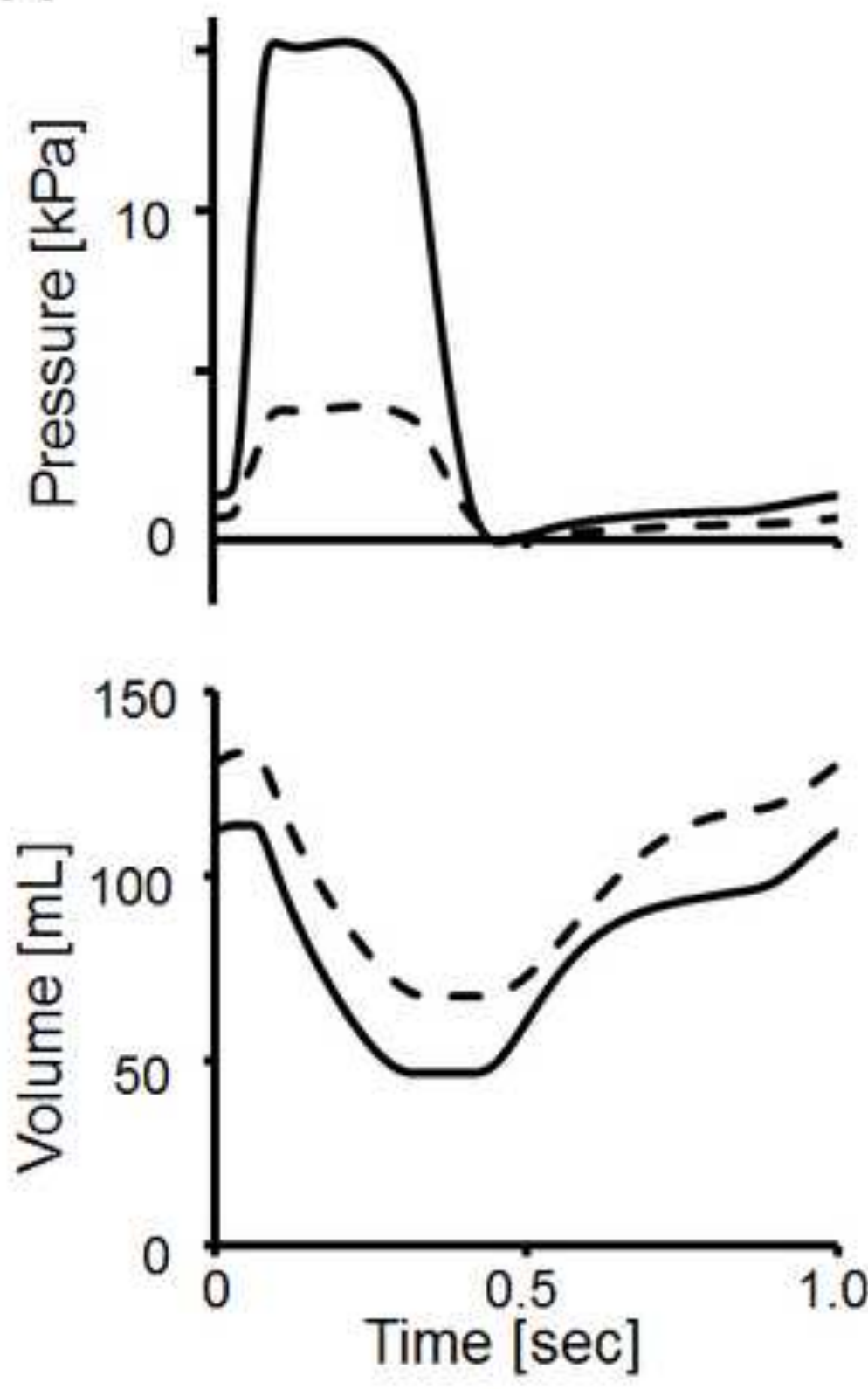

B
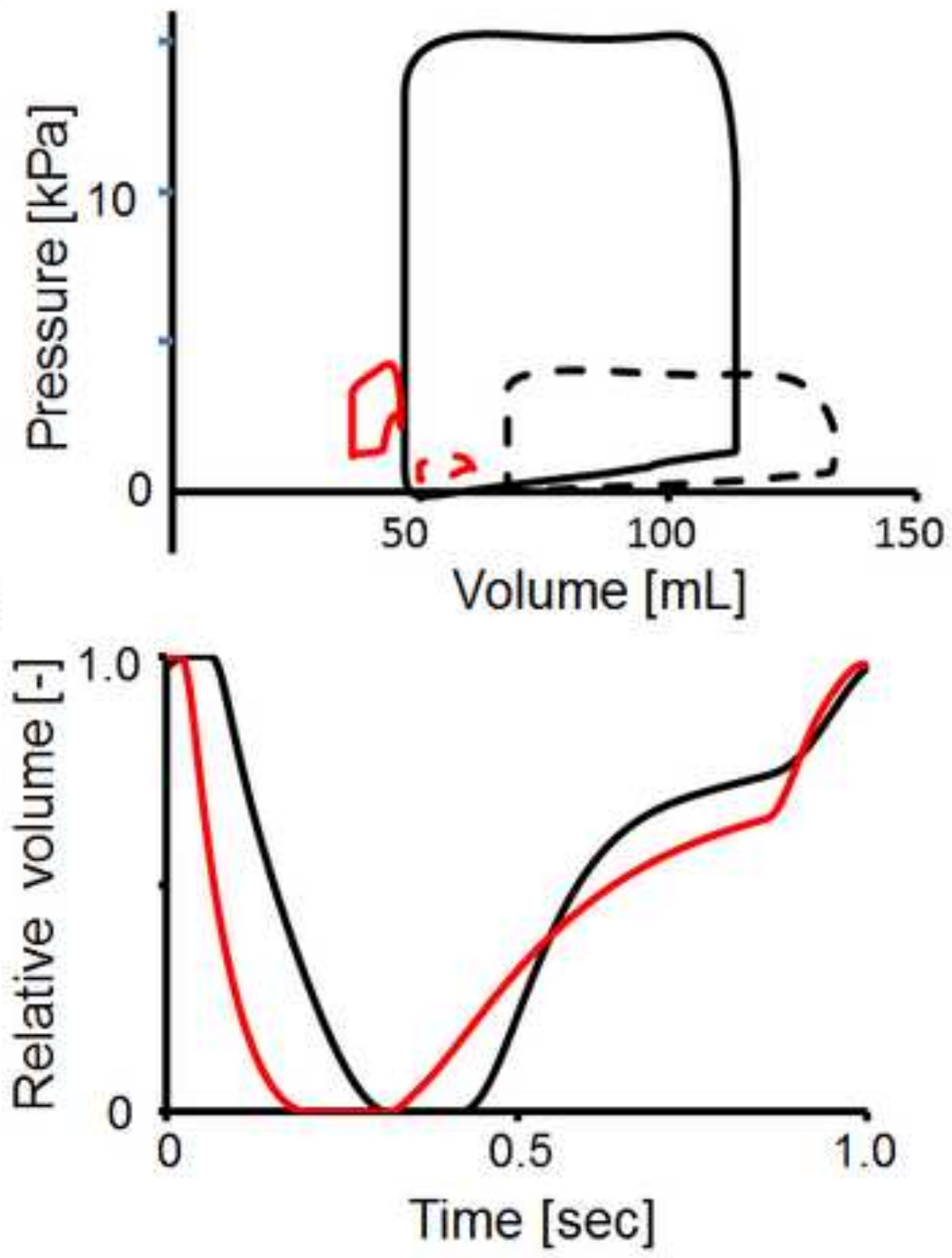

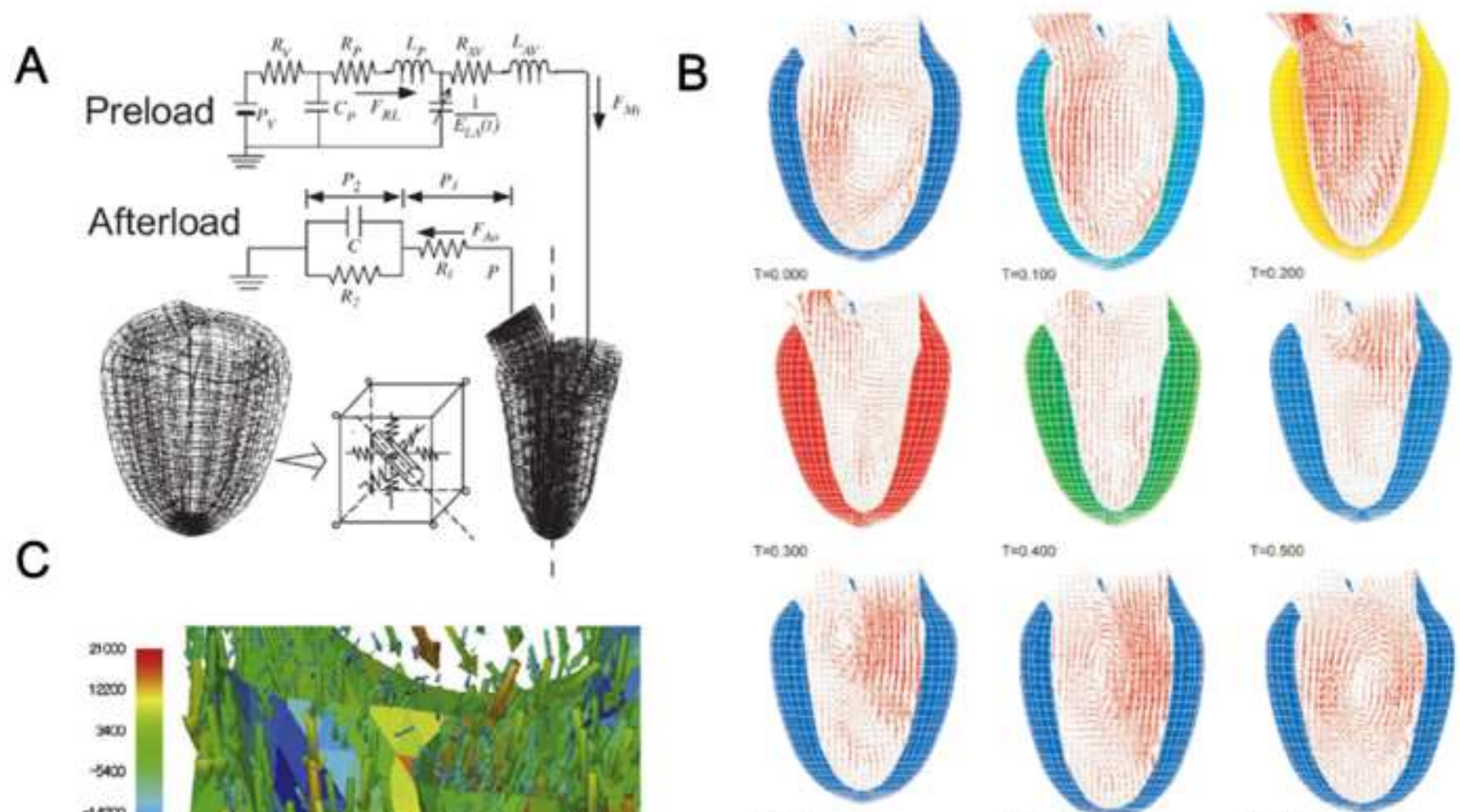

$\tan 400$
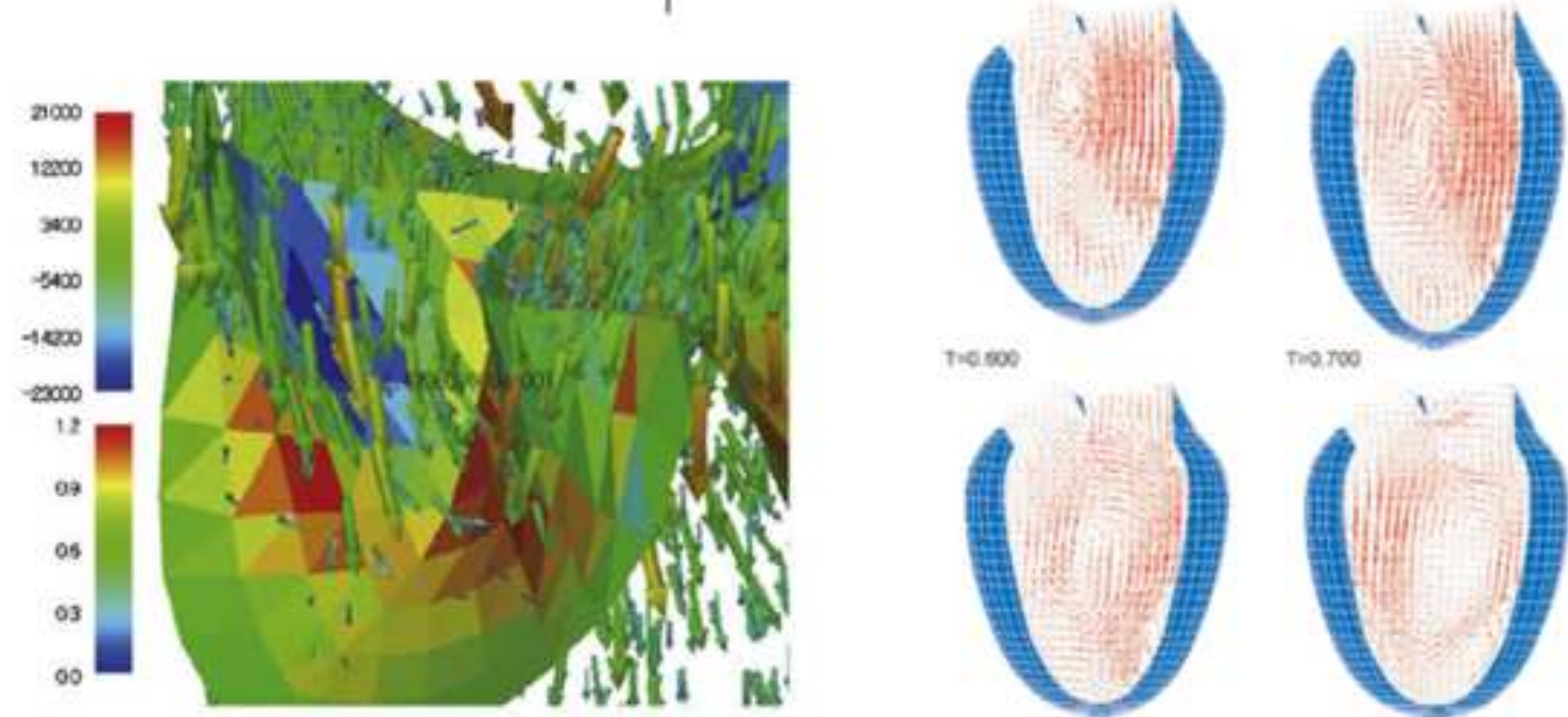

$T=0,500$

the 000

niotec
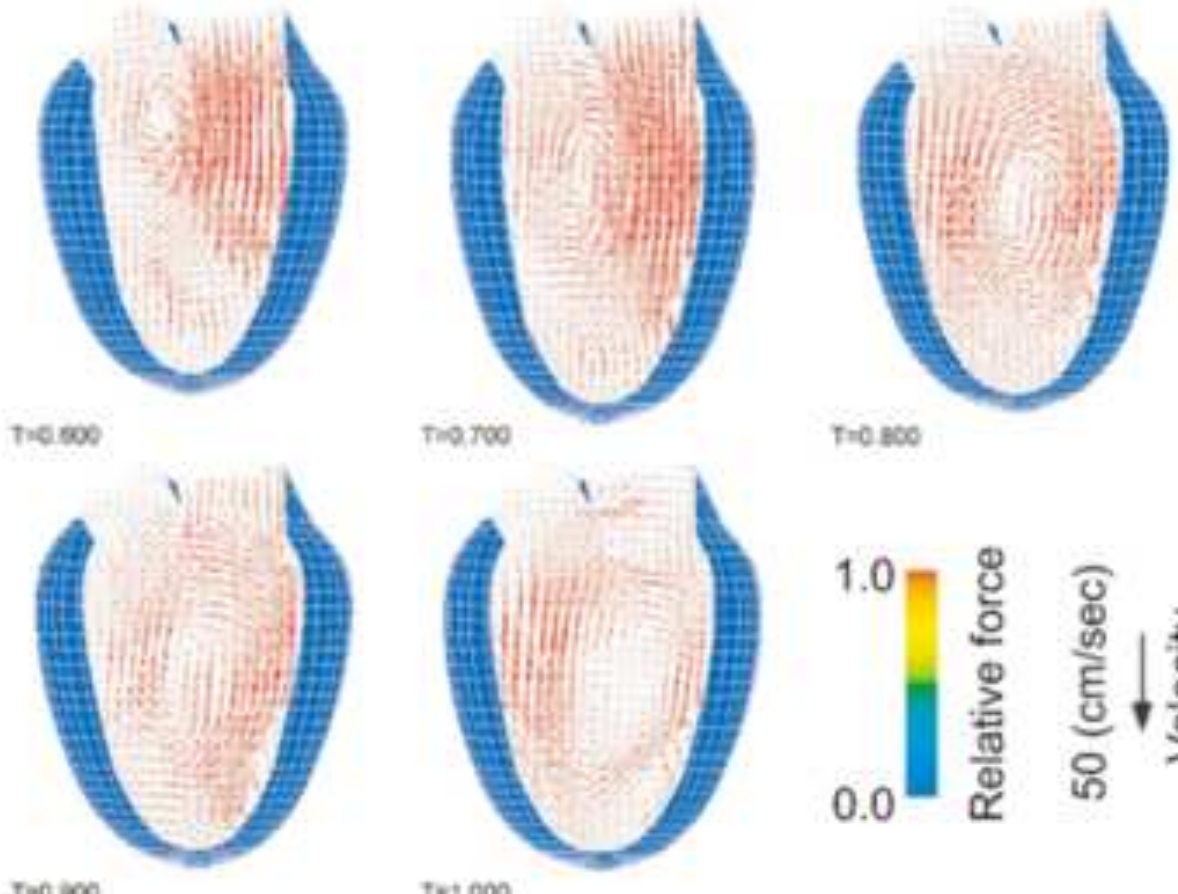






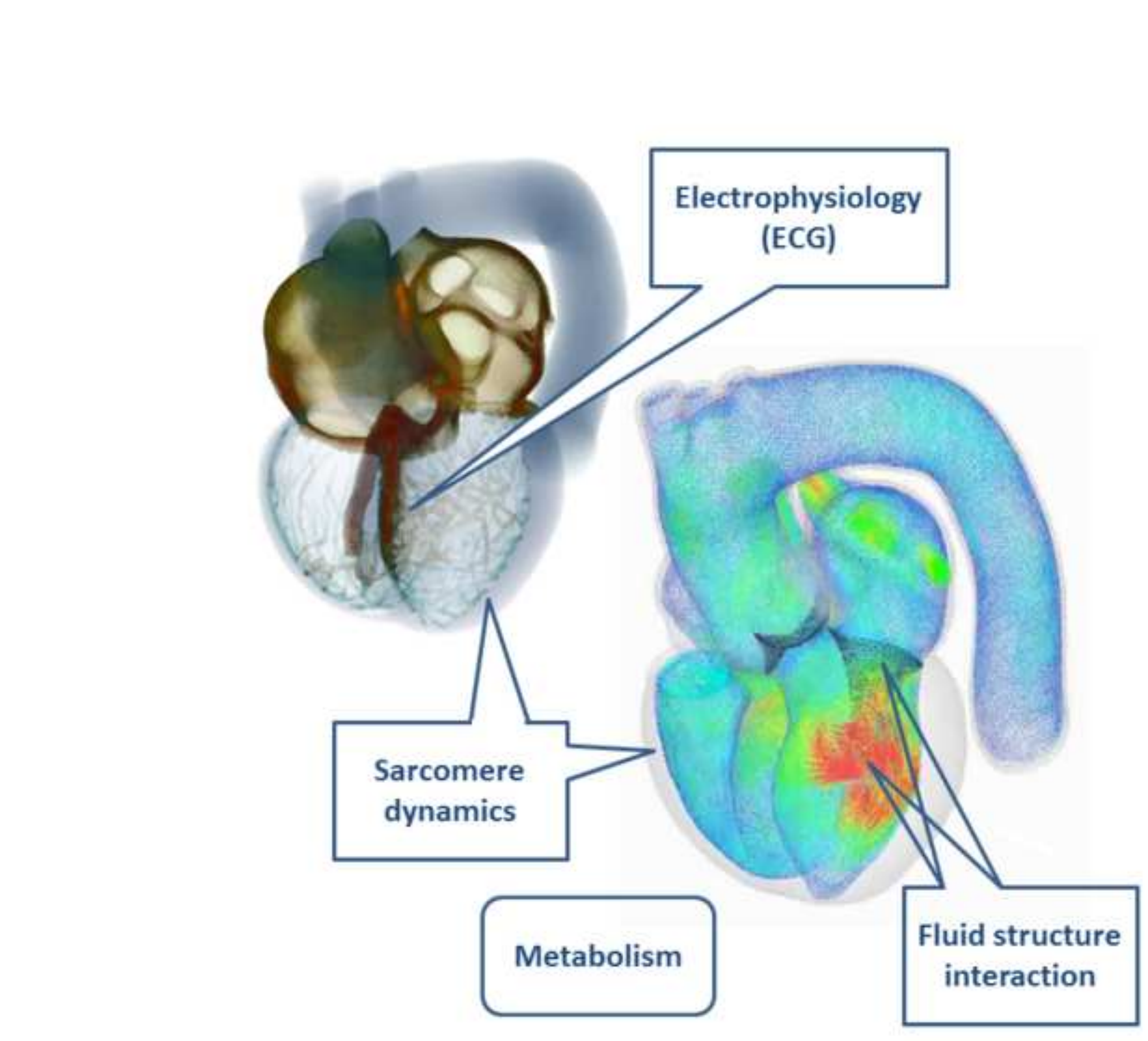

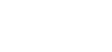

